$$
\text { "horvath" — 2007/2/16 — 12:15 — page } 391 \text { — \#1 }
$$

\title{
On four-dimensional crystallographic groups
}

\author{
ESZTER HORVÁth
}

Abstract. In his paper [12] S. S. Ryshkov gave the group of integral automorphisms of some quadratic forms (according to Dade [6]). These groups can be considered as maximal point groups of some four-dimensional translation lattices in $E^{4}$. The maximal reflection group of each point group, its fundamental domain, then the reflection group in the whole symmetry group of the lattice and its fundamental domain will be discussed. This program will be carried out first on group T. G. Maxwell [9] raised the question whether group $T$ was a reflection group. He conjectured that it was not. We proved that he had been right. We shall answer this question for other groups as well. Finally we shall give the location of the considered groups in the tables of monograph [4]. We hope that our elementary method will be useful in studying linear algebra and analytic geometry. Futhermore, 4-dimensional geometry with some visualisation helps in better understanding important concepts in higher-dimensional mathematics, in general.

Key words and phrases: four-dimensional crystallographic groups, fundamental domain, point groups, quadratic form, reflectiongroups.

ZDM Subject Classification: G40, G50, G70, H40, H60.

\section{Introduction}

The topic of geometric transformations creates connections between group theory, linear algebra and geometry. Geometric transformations form a group with successive application as the product operation. Reflections have high priority among the congruence transformations. In two- and three-dimensional space

Copyright (c) 2006 by University of Debrecen 


$$
\text { "horvath" — 2007/2/16 — 12:15 — page 392 — \#2 }
$$

every congruence transformation can be produced as a product of some reflections. When we generalize the concept of affine space, vector space and crystal structure we can draw up multidimensional geometric problems applying methods of linear algebra. The question arises in higher dimensions if we can generate some congruence groups by reflections or not. In this paper we discuss a problem in four-dimensional space which throws light on the fact that there exist transformation groups which are not reflection groups.

S. S. Ryshkov [12] gave the maximal group of integral linear automorphisms of the following quadratic form:

$$
\begin{aligned}
q(\mathbf{x})= & 4 x^{1} x^{1}+4 x^{2} x^{2}+4 x^{3} x^{3}+4 x^{4} x^{4}+2 x^{1} x^{2}-4 x^{1} x^{3}- \\
& -4 x^{1} x^{4}-4 x^{2} x^{3}-4 x^{2} x^{4}+2 x^{3} x^{4}
\end{aligned}
$$

The similar problem was discussed by Dade [6] for:

$$
\begin{aligned}
q(\mathbf{x})= & 4 x^{1} x^{1}+4 x^{2} x^{2}+4 x^{3} x^{3}+4 x^{4} x^{4}-2 x^{1} x^{2}-2 x^{1} x^{3}- \\
& -2 x^{1} x^{4}-2 x^{2} x^{3}-2 x^{2} x^{4}-2 x^{3} x^{4} \\
q(\mathbf{x})= & x^{1} x^{1}+x^{2} x^{2}+x^{3} x^{3}+x^{4} x^{4}-x^{1} x^{2}-x^{2} x^{3}-x^{3} x^{4} .
\end{aligned}
$$

This latter is equivalent [4] with the quadratic form:

$$
\begin{aligned}
q(\mathbf{x})= & x^{1} x^{1}+x^{2} x^{2}+x^{3} x^{3}+x^{4} x^{4}+x^{1} x^{2}+x^{1} x^{3}+ \\
& +x^{1} x^{4}+x^{2} x^{3}+x^{2} x^{4}+x^{3} x^{4} .
\end{aligned}
$$

Futhermore, we have the following forms:

$$
\begin{aligned}
q(\mathbf{x})= & x^{1} x^{1}+x^{2} x^{2}+x^{3} x^{3}+x^{4} x^{4}-x^{1} x^{2}-x^{3} x^{4} \\
& q(\mathbf{x})=x^{1} x^{1}+x^{2} x^{2}+x^{3} x^{3}+x^{4} x^{4} \\
q(\mathbf{x})= & x^{1} x^{1}+x^{2} x^{2}+x^{3} x^{3}+x^{4} x^{4}+x^{1} x^{2}-x^{1} x^{3}- \\
- & x^{1} x^{4}-x^{2} x^{3}-x^{2} x^{4} .
\end{aligned}
$$

This is again equivalent with the quadratic form in [4]:

$$
\begin{aligned}
q(\mathbf{x})= & x^{1} x^{1}+x^{2} x^{2}+x^{3} x^{3}+x^{4} x^{4}+x^{1} x^{2}+x^{1} x^{4}+ \\
& +x^{2} x^{3}-x^{3} x^{4}
\end{aligned}
$$

Let $\mathbf{e}_{1}, \mathbf{e}_{2}, \mathbf{e}_{3}, \mathbf{e}_{4}$ denote the basis vectors of any above lattice $\boldsymbol{\Lambda}$. The quadratic form $q(\mathbf{x})$ expresses the length square of the vector $\mathbf{x}=x^{1} \cdot \mathbf{e}_{1}+x^{2} \cdot \mathbf{e}_{2}+$ $+x^{3} \cdot \mathbf{e}_{3}+x^{4} \cdot \mathbf{e}_{4}$. G. Maxwell [9] raised the question whether the group $T$ above was a reflection group. In this paper we answer this question in the negative. Our method will be carried out in detail only for the quadratic form of $T$, however, in the tables of Section 6 we summarize the results for other cases as well. 


$$
\text { "horvath" — 2007/2/16 — 12:15 — page 393 — \#3 }
$$

\section{Basic concepts and notation}

$E$ : real affin and Euclidean space of finite dimension.

$V$ : the vector space of translations in $E$.

$B$ : symmetric, non-degenerate, positive definite bilinear form on $V$. $B$ makes $E$ above a Euclidean space. $B\left(\mathbf{v}, \mathbf{v}^{\prime}\right)$ expresses the scalar product of vectors $\mathbf{v}, \mathbf{v}^{\prime} \in \mathbf{V}$.

q: $\quad$ quadratic form on $V$. For $\mathbf{v} \in V$ put $q(\mathbf{v})=B(\mathbf{v}, \mathbf{v})$.

$|\mathbf{v}|$ : defines of the length of vector $\mathbf{v} .|\mathbf{v}|=q(\mathbf{v})^{\frac{1}{2}}$.

$H$ : hyperplane of $E$.

Definition 1. If the vector $\mathbf{n} \neq \mathbf{0}$ in $V$ is orthogonal to $H$ with respect to $B$, then the transformation:

$$
s(\mathbf{x})=\mathbf{x}-2 \cdot \frac{B(\mathbf{x}, \mathbf{n})}{B(\mathbf{n}, \mathbf{n})} \cdot \mathbf{n}, \quad \mathbf{x} \in V
$$

is called the orthogonal reflection in $H$.

The orthogonal reflection $s$ preserves $B$ and it is identity on $H$.

DEFinition 2. Groups generated by orthogonal reflections in some hyperplanes are called reflection groups or Coxeter groups.

Definition 3. Let $\mathscr{H}$ be a locally finite set of hyperplanes of $E$. $W$ denotes the group generated by the orthogonal reflections in the hyperplanes of $\mathscr{H}$. A (closed) fundamental domain of the group $W$ is a subset $C$ of $E$ if:

a) For every $\mathbf{x} \in E$, there exists $\omega \in W$ such that $\omega(x) \in C$.

b) If $\mathbf{x}, \mathbf{y} \in C$ and $\omega \in W$ are such that $\mathbf{y}=\omega(\mathbf{x})$, then $\mathbf{y}=\mathbf{x}$.

DEFinition 4. If $W$ is a group generated by the set $S$ of orthogonal reflections in the hyperplanes (walls) of a fundamental domain, then the relations of types

$$
s_{i}^{2}=1 \text { and }\left(s_{i} \cdot s_{j}\right)^{m\left(s_{i}, s_{j}\right)}=1, \quad s_{i}, s_{j} \in S
$$

determine the group. These are the defining relations of the group.

Definition 5. Let $C$ be a fundamental domain. $S$ denotes the set of orthogonal reflections in the walls of the fundamental domain $C$. The vertices of the 


$$
\text { "horvath" — 2007/2/16 — 12:15 — page 394 — \#4 }
$$

Coxeter graph represent the elements of $S$. If $m\left(s_{i}, s_{j}\right)=2$, then $s_{i}$ and $s_{j}$ are not connected, if $m\left(s_{i}, s_{j}\right) \neq 2$, then $m\left(s_{i}, s_{j}\right)$ is written on the edge connecting $s_{i}$ and $s_{j}$. When $m\left(s_{i}, s_{j}\right)=3$, then we can omit 3 from the edge.

\section{The maximal reflection group of the point group $T$}

Further on we use the former quadratic form $T$ in the form

$$
\begin{aligned}
q(\mathbf{x})= & \left(x^{1}+x^{2}-x^{3}-x^{4}\right)^{2}+\left(x^{1}-x^{3}\right)^{2}+\left(x^{1}-x^{4}\right)^{2}+ \\
& +\left(x^{2}-x^{3}\right)^{2}+\left(x^{2}-x^{4}\right)^{2}+\left(x^{1}\right)^{2}+\left(x^{2}\right)^{2}+\left(x^{3}\right)^{2}+\left(x^{4}\right)^{2} .
\end{aligned}
$$

The appropriate bilinear form is:

$$
\begin{aligned}
B(\mathbf{x}, \mathbf{y})= & 4 x^{1} y^{1}+4 x^{2} y^{2}+4 x^{3} y^{3}+4 x^{4} y^{4}+x^{1} y^{2}+x^{2} y^{1}-2 x^{1} y^{3}- \\
& -2 x^{3} y^{1}-2 x^{1} y^{4}-2 x^{4} y^{1}-2 x^{2} y^{3}-2 x^{3} y^{2}-2 x^{2} y^{4}- \\
& -2 x^{4} y^{2}+x^{3} y^{4}+x^{4} y^{3} .
\end{aligned}
$$

First we determine those hyperplane reflections that transform the lattice onto itself. Let $\mathbf{e}_{1}, \mathbf{e}_{2}, \mathbf{e}_{3}, \mathbf{e}_{4}$ be the basis vectors of the lattice and let $O$ be any point of $E$ as origin, $\overrightarrow{O E_{i}}:=\mathbf{e}_{i}(i=1,2,3,4)$. In the following we simply write plane instead of hyperplane.

Let $H_{1}$ be a possible reflection plane and let the reflection in $H_{1}$ map the origin $O$ to $O_{1}$. The vector $\overrightarrow{O O_{1}}$ belongs to the lattice $\boldsymbol{\Lambda}$. The translation by vector $\frac{1}{2} \overrightarrow{O_{1} O}$ maps $H_{1}$ onto the plane $H$ through $O$. Then the reflection in $H$ is equal to the product of the translation by vector $\overrightarrow{O_{1}}$ and the reflection in $H_{1}$. If the translation by $\frac{1}{2} \overrightarrow{O O_{1}}$ maps $H_{1}$ onto the plane $H_{2}$, then $H_{2}$ obviously is a possible reflection plane, too. Thus all the suitable reflection planes can be obtained in the following steps:

- we determine the reflection planes through the origin $O$,

- then we determine all the reflection planes by translations from the former planes.

The latter happens as follows: Let $H$ be a reflection plane through $O$ and let $\mathbf{n}=\left(n^{1}, n^{2}, n^{3}, n^{4}\right)$ be a normal vector of $H$ with respect to the scalar product derived by (2), where the greatest common divisor $\left(n^{1}, n^{2}, n^{3}, n^{4}\right)=1$. Clearly, by translations of vectors $\frac{k}{2} \cdot \mathbf{n}$ ( $k$ is an arbitrary integer $)$ we can get all the possible reflection planes.

Thus we determine all the reflection planes through the origin $O$. Let $H$ be one of these planes. Reflecting $E_{1}$ in the plane $H$ we get $E_{1}^{\prime}$. For $E_{1}^{\prime}$ it holds 
$\left|\overrightarrow{O E_{1}}\right|=\left|\overrightarrow{O E_{1}^{\prime}}\right|$, that is, by the quadratic form $\left|\overrightarrow{O E_{i}}\right|=\left|\mathbf{e}_{i}\right|=2$. If $\overrightarrow{O E_{1}^{\prime}}=\mathbf{e}_{1}^{\prime}=$ $=k^{1} \cdot \mathbf{e}_{1}+k^{2} \cdot \mathbf{e}_{2}+k^{3} \cdot \mathbf{e}_{3}+k^{4} \cdot \mathbf{e}_{4}$, then we have:

$$
\begin{gathered}
\left(k^{1}+k^{2}-k^{3}-k^{4}\right)^{2}+\left(k^{1}-k^{3}\right)^{2}+\left(k^{1}-k^{4}\right)^{2}+\left(k^{2}-k^{3}\right)^{2}+ \\
+\left(k^{2}-k^{4}\right)^{2}+\left(k^{1}\right)^{2}+\left(k^{2}\right)^{2}+\left(k^{3}\right)^{2}+\left(k^{4}\right)^{2}=4 .
\end{gathered}
$$

The sum of 9 square numbers can be equal to 4 if and only if:

- eitheir one of them is equal to 4 , the others are equal to 0 ;

- or four of the 9 numbers are equal to 1 , the others are equal to 0 .

All of $\left(k^{1}\right)^{2},\left(k^{2}\right)^{2},\left(k^{3}\right)^{2},\left(k^{4}\right)^{2}$ cannot be 0 . If one $\left(k^{i}\right)^{2}=4$, then the righthand side of $(3)$ would be 16 . Hence only the second case is possible. Thus we get the values for $k^{i}$ in Table 1 .

$$
\text { Table 1. } \mathbf{e}_{1}^{\prime}=k^{1} \cdot \mathbf{e}_{1}+k^{2} \cdot \mathbf{e}_{2}+k^{3} \cdot \mathbf{e}_{3}+k^{4} \cdot \mathbf{e}_{4}
$$

\begin{tabular}{|c|c|c|c|c|c|c|c|c|c|c|c|c|c|c|c|c|c|c|}
\hline$k^{1}$ & 1 & -1 & 1 & -1 & 0 & 0 & 0 & 0 & 0 & 0 & 0 & 0 & 0 & 0 & 1 & -1 & 1 & -1 \\
\hline$k^{2}$ & 1 & -1 & 0 & 0 & 1 & -1 & 0 & 0 & 0 & 0 & 1 & -1 & 1 & -1 & 0 & 0 & 0 & 0 \\
\hline$k^{3}$ & 1 & -1 & 0 & 0 & 0 & 0 & 1 & -1 & 0 & 0 & 0 & 0 & 1 & -1 & 0 & 0 & 1 & -1 \\
\hline$k^{4}$ & 1 & -1 & 0 & 0 & 0 & 0 & 0 & 0 & 1 & -1 & 1 & -1 & 0 & 0 & 1 & -1 & 0 & 0 \\
\hline
\end{tabular}

Then a normal vector of plane $H$ (if $E_{1} \neq E_{1}^{\prime}$ ) will be:

$$
\overrightarrow{E_{1} E_{1}^{\prime}}=\left(k^{1}-1\right) \cdot \mathbf{e}_{1}+k^{2} \cdot \mathbf{e}_{2}+k^{3} \cdot \mathbf{e}_{3}+k^{4} \cdot \mathbf{e}_{4} .
$$

We determine the normal vector $\mathbf{n}=n^{1} \cdot \mathbf{e}_{1}+n^{2} \cdot \mathbf{e}_{2}+n^{3} \cdot \mathbf{e}_{3}+n^{4} \cdot \mathbf{e}_{4}$, where $\left(n^{1}, n^{2}, n^{3}, n^{4}\right)=1$.

The possible values for $n^{i}$ are in Table 2 .

Table 2. $\mathbf{n}=n^{1} \cdot \mathbf{e}_{1}+n^{2} \cdot \mathbf{e}_{2}+n^{3} \cdot \mathbf{e}_{3}+n^{4} \cdot \mathbf{e}_{4}$

\begin{tabular}{|c|c|c|c|c|c|c|c|c|c|c|c|c|c|c|c|c|c|}
\hline$n^{1}$ & 0 & 2 & 1 & -1 & 1 & -1 & 1 & -1 & 1 & -1 & 1 & -1 & 1 & 0 & 2 & 0 & 2 \\
\hline$n^{2}$ & 1 & 1 & 0 & 1 & 1 & 0 & 0 & 0 & 0 & 1 & 1 & 1 & 1 & 0 & 0 & 0 & 0 \\
\hline$n^{3}$ & 1 & 1 & 0 & 0 & 0 & 1 & 1 & 0 & 0 & 0 & 0 & 1 & 1 & 0 & 0 & 1 & 1 \\
\hline$n^{4}$ & 1 & 1 & 0 & 0 & 0 & 0 & 0 & 1 & 1 & 1 & 1 & 0 & 0 & 1 & 1 & 0 & 0 \\
\hline
\end{tabular}

We look for those coordinates $n^{i}$, for which vectors $\mathbf{e}_{i}^{\prime}(i=1,2,3,4)$ can be expressed as an integral linear combination of $\mathbf{e}_{1}, \mathbf{e}_{2}, \mathbf{e}_{3}, \mathbf{e}_{4}$ in the reflection 
formula (4) below:

Thus

$$
\mathbf{e}_{i}^{\prime}=\mathbf{e}_{i}-\frac{2 \cdot B\left(\mathbf{e}_{i}, \mathbf{n}\right)}{B(\mathbf{n}, \mathbf{n})} \cdot \mathbf{n}
$$

$$
\frac{2 \cdot B\left(\mathbf{e}_{i}, \mathbf{n}\right)}{B(\mathbf{n}, \mathbf{n})} \cdot n^{j}
$$

must be integer for all $i=1,2,3,4$ and $j=1,2,3,4$.

By using the bilinear form (2) we shall see there exist four such $\mathbf{n}$ vectors (Table 3).

$$
\text { Table 3. } \mathbf{n}=n^{1} \cdot \mathbf{e}_{1}+n^{2} \cdot \mathbf{e}_{2}+n^{3} \cdot \mathbf{e}_{3}+n^{4} \cdot \mathbf{e}_{4}
$$

\begin{tabular}{|c|c|c|c|c|}
\hline$n^{1}$ & 0 & -1 & 1 & 1 \\
\hline$n^{2}$ & 1 & 1 & 1 & 1 \\
\hline$n^{3}$ & 1 & 0 & 0 & 1 \\
\hline$n^{4}$ & 1 & 0 & 1 & 0 \\
\hline
\end{tabular}

We still would have to examine those reflections which map $E_{1}$ onto itself.

Then we apply this method for each of the other points $E_{i}(i=2,3,4)$. So we get two more possible normal vectors. The suitable coordinates are collected in Table 4.

$$
\text { Table 4. } \mathbf{n}=n^{1} \cdot \mathbf{e}_{1}+n^{2} \cdot \mathbf{e}_{2}+n^{3} \cdot \mathbf{e}_{3}+n^{4} \cdot \mathbf{e}_{4}
$$

\begin{tabular}{|c|c|c|c|c|c|c|}
\hline$n^{1}$ & 0 & 1 & 1 & 1 & -1 & 0 \\
\hline$n^{2}$ & 1 & 0 & 1 & 1 & 1 & 0 \\
\hline$n^{3}$ & 1 & 1 & 0 & 1 & 0 & -1 \\
\hline$n^{4}$ & 1 & 1 & 1 & 0 & 0 & 1 \\
\hline
\end{tabular}

Finally we have obtained six reflections:

$\sigma_{1}$ : the reflection plane is $H_{1}$, its normal vector is $\mathbf{n}_{1}=(0,1,1,1)$,

$\sigma_{2}$ : the reflection plane is $H_{2}$, its normal vector is $\mathbf{n}_{2}=(1,0,1,1)$,

$\sigma_{3}$ : the reflection plane is $H_{3}$, its normal vector is $\mathbf{n}_{3}=(1,1,0,1)$,

$\sigma_{4}$ : the reflection plane is $H_{4}$, its normal vector is $\mathbf{n}_{4}=(1,1,1,0)$,

$\sigma_{5}$ : the reflection plane is $H_{5}$, its normal vector is $\mathbf{n}_{5}=(-1,1,0,0)$,

$\sigma_{6}$ : the reflection plane is $H_{6}$, its normal vector is $\mathbf{n}_{6}=(0,0,-1,1)$. 


$$
\text { "horvath" — 2007/2/16 — 12:15 — page 397 — \#7 }
$$

At the reflection $\sigma_{1}$ :

$$
\begin{aligned}
& \mathbf{e}_{1}^{\prime}=\mathbf{e}_{1}+\mathbf{e}_{2}+\mathbf{e}_{3}+\mathbf{e}_{4} \\
& \mathbf{e}_{2}^{\prime}=\mathbf{e}_{2} \\
& \mathbf{e}_{3}^{\prime}=-\mathbf{e}_{2}-\mathbf{e}_{4} \\
& \mathbf{e}_{4}^{\prime}=-\mathbf{e}_{2}-\mathbf{e}_{3},
\end{aligned}
$$

or by row-column multiplication:

$$
\left(\begin{array}{l}
\mathbf{e}_{1}^{\prime} \\
\mathbf{e}_{2}^{\prime} \\
\mathbf{e}_{3}^{\prime} \\
\mathbf{e}_{4}^{\prime}
\end{array}\right)=\left(\begin{array}{cccc}
1 & 1 & 1 & 1 \\
0 & 1 & 0 & 0 \\
0 & -1 & 0 & -1 \\
0 & -1 & -1 & 0
\end{array}\right) \cdot\left(\begin{array}{l}
\mathbf{e}_{1} \\
\mathbf{e}_{2} \\
\mathbf{e}_{3} \\
\mathbf{e}_{4}
\end{array}\right)
$$

by Coxeter's convention [5], or in transpose form:

$$
\left(\begin{array}{llll}
\mathbf{e}_{1}^{\prime} & \mathbf{e}_{2}^{\prime} & \mathbf{e}_{3}^{\prime} & \mathbf{e}_{4}^{\prime}
\end{array}\right)=\left(\begin{array}{llll}
\mathbf{e}_{1} & \mathbf{e}_{2} & \mathbf{e}_{3} & \mathbf{e}_{4}
\end{array}\right) \cdot\left(\begin{array}{cccc}
1 & 0 & 0 & 0 \\
1 & 1 & -1 & -1 \\
1 & 0 & 0 & -1 \\
1 & 0 & -1 & 0
\end{array}\right)
$$

or simply:

$$
\sigma_{1}:\left(\begin{array}{cccc}
1 & 0 & 0 & 0 \\
1 & 1 & -1 & -1 \\
1 & 0 & 0 & -1 \\
1 & 0 & -1 & 0
\end{array}\right)
$$

as in tables of [4].

Similarly, in this latter matrix forms:

$$
\begin{array}{r}
\sigma_{2}:\left(\begin{array}{cccc}
1 & 1 & -1 & -1 \\
0 & 1 & 0 & 0 \\
0 & 1 & 0 & -1 \\
0 & 1 & -1 & 0
\end{array}\right) \\
\sigma_{3}:\left(\begin{array}{cccc}
0 & -1 & 1 & 0 \\
-1 & 0 & 1 & 0 \\
0 & 0 & 1 & 0 \\
-1 & -1 & 1 & 1
\end{array}\right)
\end{array}
$$




$$
\text { "horvath" — 2007/2/16 — 12:15 — page 398 — \#8 }
$$

$$
\begin{gathered}
\sigma_{4}:\left(\begin{array}{cccc}
0 & -1 & 0 & 1 \\
-1 & 0 & 0 & 1 \\
-1 & 0 & 1 & 1 \\
0 & -1 & 0 & 1
\end{array}\right) \\
\sigma_{5}:\left(\begin{array}{cccc}
0 & 1 & 0 & 0 \\
1 & 0 & 0 & 0 \\
0 & 0 & 1 & 0 \\
0 & 0 & 0 & 1
\end{array}\right) \\
\sigma_{6}:\left(\begin{array}{cccc}
1 & 0 & 0 & 0 \\
0 & 1 & 0 & 0 \\
0 & 0 & 0 & 1 \\
0 & 0 & 1 & 0
\end{array}\right)
\end{gathered}
$$

It is easy to verify that:

$$
\begin{aligned}
\sigma_{5} & =\sigma_{2} \cdot \sigma_{1} \cdot \sigma_{2} \\
\sigma_{6} & =\sigma_{4} \cdot \sigma_{3} \cdot \sigma_{4} \\
\left(\sigma_{1} \cdot \sigma_{2}\right)^{3} & =\left(\sigma_{3} \cdot \sigma_{4}\right)^{3}=I .
\end{aligned}
$$

( $I$ denotes the identity map).

Thus the reflection subgroup, mapping $O$ onto itself, can be generated by $\sigma_{1}, \sigma_{2}, \sigma_{3}, \sigma_{4}$. The group generated by $\sigma_{1}$ and $\sigma_{2}$ above is denoted by $A_{2}$ (see the notation in [1]) and the same holds for $\sigma_{3}$ and $\sigma_{4}$ as well. Therefore the maximal reflection subgroup of the point group of $T$ can be denoted as a direct product $A_{2} \times A_{2}$, since $H_{1}, H_{2} \perp H_{3}, H_{4}$. The Coxeter graph of this group is shown in Figure 1.

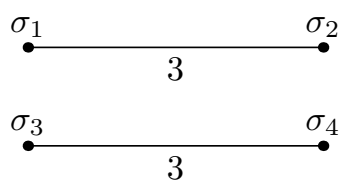

Figure 1. The Coxeter graph of the maximal reflection subgroup of the point group of $T$ 


$$
\text { "horvath" — 2007/2/16 — 12:15 — page 399 — \#9 }
$$

\section{The point group of $T$ is not a reflection group}

We determine the fundamental domain of $A_{2} \times A_{2}$ above, that is $F_{A_{2} \times A_{2}}$. This can be obtained as the intersection of suitable half spaces determined by planes $H_{1}, H_{2}, H_{3}, H_{4}$, respectively. The inequalities below determine the points $\mathbf{x}=\left(x^{1}, x^{2}, x^{3}, x^{4}\right)$ of the fundamental domain:

$$
\begin{aligned}
& H_{1}^{+}: \quad-x^{1}+x^{3}+x^{4} \geq 0 \quad\left(B\left(\mathbf{x}, \mathbf{n}_{1}\right) \geq 0\right), \\
& H_{2}^{+}: \quad x^{2}-x^{3}-x^{4} \geq 0 \quad\left(B\left(\mathbf{x},-\mathbf{n}_{2}\right) \geq 0\right) \text {, } \\
& H_{3}^{+}: \quad x^{1}+x^{2}-x^{3} \geq 0 \quad\left(B\left(\mathbf{x}, \mathbf{n}_{3}\right) \geq 0\right) \text {, } \\
& H_{4}^{+}: \quad-x^{1}-x^{2}+x^{4} \geq 0 \quad\left(B\left(\mathbf{x},-\mathbf{n}_{4}\right) \geq 0\right) \text {. }
\end{aligned}
$$

The intersection line of planes $H_{i}, H_{j}, H_{k}$ will be denoted by $f_{i j k}$. The direction vectors of $f_{123}, f_{124}, f_{134}, f_{234}$ are $(1,1,2,-1),(1,1,-1,2),(2,-1,1,1)$, $(-1,2,1,1)$, respectively. Using these we get the 2 -dimensional faces of the fundamental domain. $A_{i j}$ denotes the intersection of $H_{i}$ and $H_{j}$ (Figure 2).
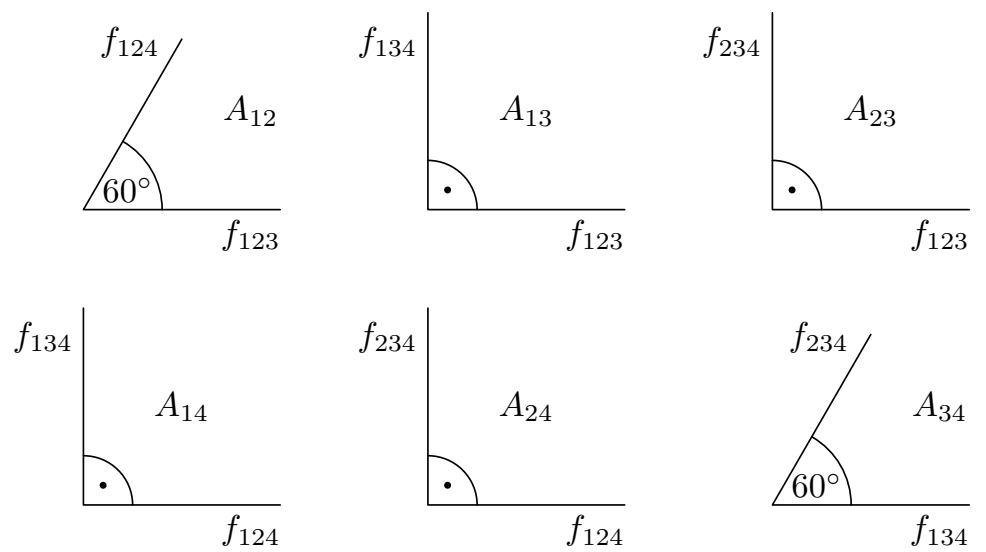

Figure 2. The 2-dimensional faces of the fundamental domain of the maximal reflection subgroup of the point group of $T$

We still have to detect whether the fundamental domain can be mapped onto itself by other symmetry operations or not. Of course, we look for those transformations which map the lattice onto itself as well.

Further on we give such transformations: 


$$
\text { "horvath" — 2007/2/16 — 12:15 — page } 400 \text { — \#10 }
$$

(1) Transformation $f_{1}$ maps the half space $H_{1}^{+}$onto $H_{3}^{+}$, and $H_{2}^{+}$onto $H_{4}^{+}$,

$$
f_{1}:\left(\begin{array}{cccc}
0 & 0 & 1 & 0 \\
0 & 0 & 0 & 1 \\
1 & 0 & 0 & 0 \\
0 & 1 & 0 & 0
\end{array}\right)
$$

$f_{1} \cdot f_{1}=I . f_{1}$ is a reflection in a 2 -dimensional plane.

(2) Transformation $f_{2}$ maps the half space $H_{1}^{+}$onto $H_{4}^{+}$, and $H_{2}^{+}$onto $H_{3}^{+}$,

$$
f_{2}:\left(\begin{array}{cccc}
0 & 0 & 0 & -1 \\
0 & 0 & -1 & 0 \\
0 & -1 & 0 & 0 \\
-1 & 0 & 0 & 0
\end{array}\right)
$$

$f_{2} \cdot f_{2}=I . f_{2}$ is a reflection in a 2 -dimensional plane.

(3) Transformation $f_{1} \cdot f_{2}=f_{2} \cdot f_{1}$ :

$$
\left(\begin{array}{cccc}
0 & -1 & 0 & 0 \\
-1 & 0 & 0 & 0 \\
0 & 0 & 0 & -1 \\
0 & 0 & -1 & 0
\end{array}\right)
$$

By the Coxeter graph it would be possible to have a transformation which maps the half space $H_{1}^{+}$into $H_{2}^{+}$and the half spaces $H_{3}^{+}$and $H_{4}^{+}$are stable under this transformation. Howewer, this would be a reflection in a three-dimensional plane bisecting the angle of $H_{1}^{+}$and $H_{2}^{+}$. But we have already found all convenient reflections, it means that this reflection does not map the lattice onto itself. Similarly there is no such transformation which maps the half space $\mathrm{H}_{3}^{+}$ into $H_{4}^{+}$and leaves $H_{1}^{+}$and $H_{2}^{+}$invariant. After all there are no more such transformations.

Thus the elements of group $F=\left\{1, f_{1}, f_{2}, f_{1} \cdot f_{2}\right\}$ in point group $T$ map both the fundamental domain of the maximal reflection subgroup and the lattice onto itself. Clearly, all elements of $T$ can uniquely be obtained as a product $f \cdot d$, where $f \in F$ and $d \in A_{2} \times A_{2}$. The reason of this is the following: Let the transformation $t \in T$ map the point $P$ into $P^{\prime}$. Let $P$ be a point of the fundamental domain $F_{1}$ and $P^{\prime}$ in the fundamental domain $F_{2}$. By product of some reflections we can get $F_{1}$ from $F_{2}$. Then the image of $P^{\prime}$ is the point $P_{1}$. Thus there is a transformation $f \in F$ which maps the point $P$ onto $P_{1}$. Hence the transformation $t$ is the product of $f$ and some reflections. $F$ has 4 elements, $A_{2} \times A_{2}$ has $6 \cdot 6=36$ elements, so the order of the point group $T$ is $4 \cdot 36=144$. 


\section{The maximal reflection subgroup}

We shall determine the maximal reflection subgroup in the whole symmetry group of the lattice $\boldsymbol{\Lambda}$ of $T$ as follows.

The fundamental domain $F^{*}$ of the reflection subgroup will be a polyhedron which is a subset of $F_{A_{2} \times A_{2}}$. The group will be generated by reflections in the 3-dimensional walls of $F^{*}$. Therefore we get the fundamental domain $F^{*}$ in the following steps:

- We shift the plane $H_{i}$ by the vector $\frac{1}{2} \underline{n}_{i}$ or $-\frac{1}{2} \underline{n}_{i}(i=1,2,3,4,5,6)$, the image of $H_{i}$ will be denoted by $H_{i^{\prime}}$. We choose the plane that intersects the corner domain $F_{A_{2} \times A_{2}}$.

- We choose the half spaces above that contain the origin $O$.

- The intersection of these half spaces and $F_{A_{2} \times A_{2}}$ will be determined.

The inequalities below determine the fundamental domain $F^{*}$ :

$$
\begin{array}{rrr}
H_{1}^{+}: & -x^{1}+x^{3}+x^{4} \geq 0, \\
H_{2}^{+}: & x^{2}-x^{3}-x^{4} \geq 0, \\
H_{3}^{+}: & x^{1}+x^{2}-x^{3} \geq 0, \\
H_{4}^{+}: & -x^{1}-x^{2}+x^{4} \geq 0, \\
H_{5^{\prime}}^{+}: & x^{1}-x^{2}+1 \geq 0, \\
H_{6^{\prime}}^{+}: & x^{3}-x^{4}+1 \geq 0 .
\end{array}
$$

A 2-dimensional axonometric projection of the fundamental domain is shown in Figure 4. $B_{i j k l}$ denotes the common point of the corresponding planes $H_{i}, H_{j}, H_{k}$, $H_{l}$. The maximal reflection subgroup in the whole symmetry group of the lattice $\boldsymbol{\Lambda}$ is generated by reflections $\sigma_{1}, \sigma_{2}, \sigma_{3}, \sigma_{4}, \sigma_{5^{\prime}}, \sigma_{6^{\prime}}$ in planes $H_{1}, H_{2}, H_{3}, H_{4}, H_{5^{\prime}}$, $H_{6^{\prime}}$, respectively. The Coxeter graph of this group is shown in Figure 3. It is the group denoted by $\tilde{A}_{2} \times \tilde{A}_{2}$ (see the notation in [1]).
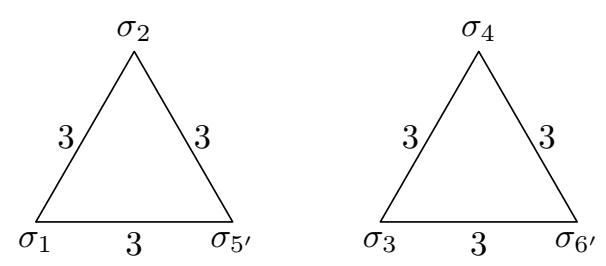

Figure 3. The Coxeter graph of the maximal reflection group of the whole symmetry group of the lattice $\boldsymbol{\Lambda}$ 


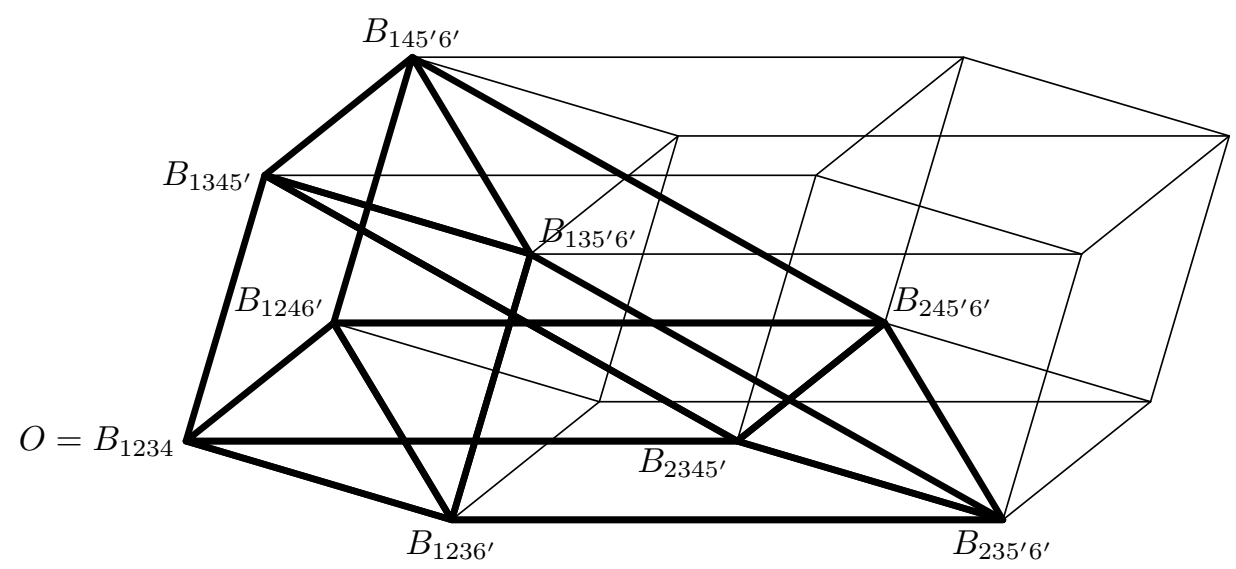

Figure 4. The 2-dimensional axonometric projection of the fundamental domain $F^{*}$

\section{Results for other cases}

We apply the method above for each quadratic form. Table 5 and Table 6 contain the data for all mentioned cases. The location in monograph [4] (see its tables) gives the crystal family, the crystal system, the Q-class, and the Z-class of the groups by their numberings.

Table 5. Results for the different cases I. (see $[1,4]$ )

\begin{tabular}{|l|c|c|c|}
\hline $\begin{array}{l}\text { The group in } \\
\text { Introduction }\end{array}$ & $T$ & $P_{4}$ & $S_{4}$ \\
\hline $\begin{array}{l}\text { The maximal reflection } \\
\text { group of point group }\end{array}$ & $A_{2} \times A_{2}$ & $A_{4}$ & $A_{4}$ \\
\hline $\begin{array}{l}\text { Is the group a pure } \\
\text { reflection group? }\end{array}$ & NO & NO & NO \\
\hline $\begin{array}{l}\text { The location in the } \\
\text { tables of monograph [4] }\end{array}$ & XXI.29/09/01 & XXII.31/07/01 & XXII.31/07/02 \\
\hline $\begin{array}{l}\text { The reflection subgroup } \\
\text { in the whole symmetry } \\
\text { group }\end{array}$ & $\tilde{A}_{2} \times \tilde{A}_{2}$ & $\tilde{A}_{4}$ & $\tilde{A}_{4}$ \\
\hline
\end{tabular}




$$
\text { "horvath" — 2007/2/16 — 12:15 — page } 403 \text { — \#13 }
$$

On four-dimensional crystallographic groups

Table 6. Results for the different cases II. (see $[1,4]$ )

\begin{tabular}{|l|c|c|c|}
\hline $\begin{array}{l}\text { The group in } \\
\text { Introduction }\end{array}$ & $B$ & $C_{4}$ & $Q_{4}$ \\
\hline $\begin{array}{l}\text { The maximal reflection } \\
\text { group of point group }\end{array}$ & $G_{2} \times G_{2}$ & $C_{4}$ & $F_{4}$ \\
\hline $\begin{array}{l}\text { Is the group a pure } \\
\text { reflection group? }\end{array}$ & NO & YES & YES \\
\hline $\begin{array}{l}\text { The location in the } \\
\text { tables of monograph [4] }\end{array}$ & XXI.30/13/01 & XXIII.32/21/01 & XXIII.33/16/01 \\
\hline $\begin{array}{l}\text { The reflection subgroup } \\
\text { in the whole symmetry } \\
\text { group }\end{array}$ & $\tilde{G}_{2} \times \tilde{G}_{2}$ & $\tilde{C}_{4}$ & $\tilde{F}_{4}$ \\
\hline
\end{tabular}

\section{References}

[1] N. Bourbaki, Groupes et Algébres de Lie. Chap. IV-VI, Hermann, Paris, 1968, Russian translation Mir, Moscow, 1972, English translation Springer, 2002.

[2] V. A. Bovdi, P. M. Gudovik, V. P. Rudko, Torsion-free Groups with indecomposable Holonomy Group I., J. Group Theory 5, no. 1 (2002), 75-96.

[3] V. A. Bovdi, P. M. Gudovik, V. P. Rudko, Torsion-free Groups with indecomposable Holonomy Group II., J. Group Theory 7, no. 4 (2004), 555-569.

[4] H. Brown, R. Bülow, J, Neubüser, H. Wondratschek, H. Zassenhaus, Crystallographic Groups of Four-dimensional Space, Wiley Interscience, 1978.

[5] H. S. M. Coxeter, W. O. J. Moser, Generators and Relations for Discrete Groups, 4th ed., Ergebnisse der Math. und ihrer Grenzgebiete, Bd. 14, Springer Verlag, Berlin-Heidelberg-New York, 1980.

[6] E. C. Dade, The maximal finite groups of $4 \times 4$ integral matrices, Illinois J. Math. 9 (1965), 99-122.

[7] G. M. Martin, Transformation Geometry, Springer Verlag, New York-Heidelberg-Berlin, 1982.

[8] E. Horváth, On a fundamental theorem of reflection geometry, Annales Univ. Sci. Budapest 46 (2003), 133-148.

[9] G. Maxwell, The crystallography of Coxeter groups, J. Algebra 35 (1975), 159-178.

[10] E. Molnár, Some old and new aspects on the crystallographic groups, Periodica Polytechnica Ser. Mech. Eng 36 (1992), 191-218. 


$$
\text { "horvath" — 2007/2/16 — 12:15 — page } 404 \text { — \#14 }
$$

[11] E. Molnár, I. Prok, J. Szirmai, D-V Cells and Fundamental Domain for Crystallographic Groups, Algorithms, and Graphic Realizations, Mathematical and Computer Modelling 38, no. 7-9, Hungarian Applied Mathematics and Computer Applications, Guest editor: A. Benczur (2003), 929-943, ww. elsevier.com/locate/mcm.

[12] S. S. Ryshkov, Maximal finite groups of integral $n \times n$ matrices and full groups of integral authomorphisms of positive quadratic forms (Bravais models), Trudy Mat. Inst. Steklov 128 (1972), 183-211 (in Russian), Proc. Steklov Inst. Math. 128 (1972), 217-250 (in English).

[13] S. S. Ryshkov, On complete groups of integral automorphisms of quadratic forms, Soviet. Math. Dokl. 13 (1972), 1251-1254.

ESZTER HORVÁTH

SZILÁGYI ERZSÉBET SECONDARY SCHOOL

MÉSZÁROS U. 5-7.

H-1016 BUDAPEST

HUNGARY

E-mail: horveszt@t-online.hu

(Received April, 2006) 